\title{
An illusory depth reversal dependent upon rotary motion
}

\author{
EUGENE R. WIST and BEATRICE E. HUNT \\ Franklin and Marshall College, Lancaster, Pennsylvania 17603
}

\begin{abstract}
A textured rotating disk viewed obliquely was found to perceptually reverse its orientation in depth under a variety of conditions, none of which were able to eliminate the illusory depth reversal. The only necessary conditions for its production appear to be that some texture be present, that the stimulus be moving, and that the stimulus be viewed from an oblique angle. Qualitative observations indicated that changes in the perceived size and speed of the texture elements of the rotating stimulus consistent with constancy mechanisms for size and speed accompanied changes in apparent depth orientation.
\end{abstract}

In order to demonstrate the phenomenon of rollvection, an apparatus was constructed like that described by Dichgans, Diener, and Brandt (1974), consisting of a white disk, $100 \mathrm{~cm}$ in diameter, over which blue or red dots, $1.9 \mathrm{~cm}$ in diameter, were distributed. This disk was rotated by means of a variable-speed motor at right angles to the subject's median plane, $30 \mathrm{~cm}$ from the eyes, to induce the sensation of self-motion around the line of sight. Quite accidentally, it was discovered that when this disk was viewed monocularly from a distance of about $100 \mathrm{~cm}$ at an angle of about $30^{\circ}$ to the median plane, a very compelling illusory depth reversal resulted. As shown in Figure 1 (Panel A), instead of perceiving the rotating disk in its true orientation, slanting away to the left (A1), it is seen periodically in the reversed orientation slanting away to the right (A2).

Obviously, this is yet another example of a multistable figure (Attneave, 1971). Certain of its properties, however, make it especially worth reporting: (1) Motion of the disk appears to be necessary in order to induce the depth reversal. We have rarely gotten reports of such reversals when the disk was not in motion, and these have resulted only after the disk had been observed in motion. Paradoxically, stimulus motion appears to be necessary for the induction of this illusion, in spite of the fact that the gradient of motion across the retina is consistent with the true and not the illusory depth orientation. It ought to contribute to the veridical perception of depth orientation but doesn't. (2) It is a very robust effect: no subject has ever failed to observe it, and none of the stimulus variations we explored were able to completely elininate it.

This study constituted the senior research project of the second author. Thanks are due to $\mathbf{R}$. Abessinio and R. Steere for construction of the apparatus and to Charles Eldredge for making the figure.
The purpose of this exploratory study was to determine the effect of various stimulus and fixation conditions upon the magnitude of this illusion.

\section{METHOD}

\section{Subjects}

A total of 41 undergraduate students, 19 male and 22 female, served as subjects. Of this number, 20 received laboratory credit for an introductory psychology course. No screening tests were employed.

\section{Apparatus}

A variable-speed reversible motor was used to rotate a reinforced $1 / 4-i n$. plywood disk, $100 \mathrm{~cm}$ in diameter, whose front surface was painted flat white and covered with 90 red and 90 blue colored, randomly distributed paper disks, $1.9 \mathrm{~cm}$ in diameter. These colcred disks will henceforth be referred to as "dots," and the $100-\mathrm{cm}$ disk which contained them as the standard disk. The standard disk was centered just in front of and parallel to a black velveteen background, $210 \mathrm{~cm}$ high and $320 \mathrm{~cm}$ wide, was viewed from a distance of $183 \mathrm{~cm}$, and was slanted with its right edge nearer at an angle of $32^{\circ}$ to the subject's median plane. At this distance and viewing angle, it subtended a visual angle of $30^{\circ}$ vertically and $10^{\circ}$ horizontally. Illumination of the rotating stimulus, unless otherwise specified, was provided by a 150 -W reflector flood light placed at $183 \mathrm{~cm}$ from the disk at right angles to its plane of rotation. At this distance, the luminance of the white surface of the disk as measured from the subject's viewing position was $22 \mathrm{~mL}$.

\section{Procedure}

The subjects were assigned alternately to one of two groups in the order of their appearance at the laboratory. Each subject viewed the disk monocularly with his or her right eye. The left eye was covered with an eye patch.

Group $1(\mathrm{~N}=20)$ subjects were tested individually under the following conditions.

Fixation vs. ocular pursuit. The standard disk was rotated clockwise at $108^{\circ} / \mathrm{sec}(18 \mathrm{rpm})$, with fixation either ufon its center or upon a point located $1.5^{\circ}$ to the left of its far ecige or $1.5^{\circ}$ to the right of its near edge. For the ocular pursuit condition, the subjects tracked a fluorescent orange dot on the perimeter of the disk; it was of the same size as the other dots, but had a smaller, black dot at its center. These conditions are illusirated in Panel D1 of the Figure 1. 


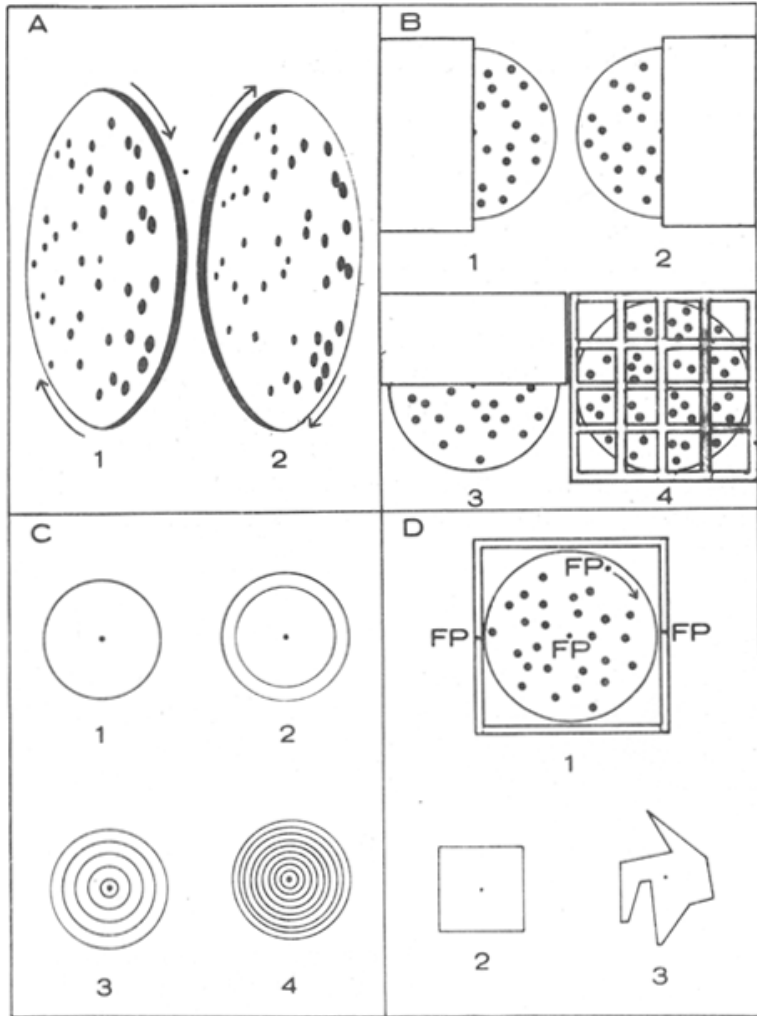

Figure 1. Stimuli used in the experiments (not drawn to scale). (A) (1) The standard disk shown in its actual orientation, and (2) in its illusory orientation. (B) Occlusion of the standard disk: (1) left half, (2) right half, and (3) top half occluded. (4) Occlusion by a grid. (C) Concentric rings conditions: (1) no ring, (2) one ring, (3) four rings, (4) eight rings. (D) (1) Fixation vs. ocular pursuit. The lower points labeled $F P$ represent fixation to the left (far side) of the center, and to the right (near side) of the disk. The point and arrow at the top represent the ocular pursuit condition. (2) and (3) Examples of noncircular stimuli which produced depth orientation reversals when rotated: (2) a $10-\mathrm{cm}$ square, and (3) an irregular polygon whose longest dimension is $17.5 \mathrm{~cm}$.

Occlusion of the disk. The standard disk was rotated at the same speed and in the same direction as in the preceding condition, with fixation at its center. As shown in the figure (Panel B1, 2, and 3), the left, right, or top half of the disk was occluded by a sheet of black composition board attached $5 \mathrm{~cm}$ in front of the disk to the frame illustrated in Panel D1. In addition, a fourth viewing condition consisted of the grid shown in Panel B4. The vertical and horizontal bars of this grid were $2.5 \mathrm{~cm}$ wide; they had $30.5-\mathrm{cm}$ separations and were positioned on the frame $5 \mathrm{~cm}$ in front of and parallel to the disk.

Flicker. This condition differed from the above condition in that the disk was not occluded. Instead, it was illuminated with a Grass PS2 photostimulator located immediately adjacent to the flood lamp at either 10 or $17.4 \mathrm{~Hz}$. The luminance of the disk measured at the latter frequency with a Spectra photometer was $3.2 \mathrm{~mL}$. These frequencies were selected because of the effect they had upon the perceived motion of the dots distributed over the surface of the disk. Both flicker rates produced the impression of a slower rate of rotation, of a random motion of some dots while other dots appeared to rotate about centers other than the true center of the disk, and of retrograde motion of the dots near the disk's perimeter.
Group $2(\mathbf{N}=21)$ subjects were tested individually under the following conditions:

Speed and direction. The center of the standard disk was fixated while the disk rotated either clockwise or counterclockwise at 26 $(4.3 \mathrm{rpm})$ or $108^{\circ} / \mathrm{sec}$.

Concentric rings. Four smaller disks were employed; these were $50 \mathrm{~cm}$ in diameter, were painted flat white, and contained 0,1 , 4 , or 8 black $3-\mathrm{mm}$ concentric rings as shown in Panel $\mathrm{C}$ of the figure. Stimulus speed was $108^{\circ} / \mathrm{sec}$ clockwise, and fixation was maintained in the center of each disk.

The order of conditions for both groups of subjects was randomized. The order of presentation of the stimuli within conditions was also randomized. Each subject received a total of three 1-min trials separated by 15 -sec rest periods for each stimulus. A rest period of approximately $2 \mathrm{~min}$ occurred between trial blocks.

\section{Measures of Changes in Depth Orientation}

Subjects used a hand-held pushbutton to indicate reversals of the depth orientation of the rotating disk. The button was depressed to indicate an apparent reversal in orientation and released to indicate a return to the actual orientation. Latency to the first reversal was measured by an electric timer which started when the eye was opened just after the disk was set in motion and stopped when the pushbutton was depressed. Depression of the button also advanced a cumulative counter by one count in order to record the number of reversals per trial. A second timer measured the total duration of button depression per minute and thus provided a measure of the total duration of the illusory depth orientation per trial.

At the beginning of the experiment, the subject was seated on a stool with a backrest adjusted in height so that the center of the disk was at eye level. The subject was then instructed in the use of the pushbutton, and several practice trials were given with monocular fixation of the standard disk rotating clockwise at $108^{\circ} / \mathrm{sec}$

\section{RESULTS AND DISCUSSION}

Individual means were calculated for each block of three trials for each condition for each of the three measures. In addition, a fourth measure was obtained for each subject. The total duration of the illusory depth orientation for a given 1-min trial was divided by the number of reversals during that trial to obtain a measure of the mean duration in seconds of illusory depth orientation per reversal.

In the interest of clarity, the Table 1 gives only those means which were significantly different from those for the standard disk $\left(108^{\circ} / \mathrm{sec} \mathrm{cw}\right)$ in the top row. $t$ tests for related means were used for these comparisons. Groups 1 and 2 did not differ significantly for the standard disk. Therefore, their results are combined in the table. ${ }^{1}$

A strong depth reversal is indicated by a short latency to the first reversal, a low reversal rate, a large total illusion duration, and $D / R$ ratio. It was not expected that all four measures would necessarily be highly correlated, since, for example, a low reversal rate might reflect either a tendency to perceive the disk in its actual or in the reversed orientation for most of the 1-min trial period.

It is clear from the table that the measure least sensitive to differences in stimulus conditions was the 
Table 1

Means of Four Measures of Depth Orientation Reversals as a Function of Stimulus Orientation

\begin{tabular}{lcccc}
\hline \multicolumn{1}{c}{$\begin{array}{c}\text { Stimulus } \\
\text { Condition }\end{array}$} & $\begin{array}{c}\text { Latency } \\
\text { (seconds) }\end{array}$ & $\begin{array}{c}\text { Number of } \\
\text { Reversals } \\
\text { (minutes) }\end{array}$ & $\begin{array}{c}\text { Total } \\
\text { Duration } \\
\text { (seconds) }\end{array}$ & D/R \\
\hline Standard Disk & & & & \\
$\quad \begin{array}{l}\text { Center fixation } \\
\text { Ocular pursuit }\end{array}$ & 9.75 & 4.89 & 31.54 & 13.64 \\
$\begin{array}{l}\text { Fixation on right } \\
\text { Occlusion by grid } \\
\text { Left half occluded }\end{array}$ & $18.06^{*}$ & & & $6.69^{*}$ \\
$\quad \begin{array}{llll}\text { Flicker } \dagger \\
\text { Small Disk }\end{array}$ & $28.60^{* *}$ & $2.53^{*}$ & $16.80^{* *}$ & $10.08^{*}$ \\
$\quad$ No rings & & & $19.51^{* *}$ & \\
$\quad$ Eight rings & $24.00^{* *}$ & & & \\
\hline
\end{tabular}

tThe data for the 10- and 17.4-Hz conditions were combined since they were not statistically different.

${ }^{*} p<.05$

$* * p<.01$

number of reversals in depth orientation per minute (R). The only significant difference obtained with this measure was for the condition in which the left half of the standard disk was occluded. Latency to the first reversal of depth orientation $(L)$ was the most sensitive measure, since six of the stimulus conditions resulted in significant latency increases. A different pattern of significant effects is shown for the total duration of reversed depth orientation per 1-min trial (D) and the D/R measures. The latter was more affected by conditions in the upper half, while the former was more affected by conditions in the lower half of the table. Only when the left half of the disk was occluded did all four measures differ significantly from the corresponding measures obtained for the standard disk.

The most remarkable feature of these data is the fact that none of the conditions eliminated the illusion. Even the half-disks illustrated in Panel B of the figure yielded reversals in depth orientation. The only effective way of eliminating this illusion we have found to date is to keep it stationary, or nearly so, or present it in a frontal, or near frontal, orientation. Furthermore, in an unpublished study conducted with 25 different subjects, it was found that reversals in depth orientation also occur with stimulus shapes such as those illustrated in Panels D2 and 3 of the figure, as well as with smaller disks, 10 and $15 \mathrm{~cm}$ in diameter, which were either plain or covered with similar or lower densities of colored dots and viewed at the same distance and orientation as that of the standard disk (Hunt, Progin, \& Stone, Note 1).

\section{Qualitative Observations}

We observed three additional phenomena associated with illusory depth reversals of the standard disk which are noteworthy. First, during a reversal, the disk may not appear to be flat, but instead to be noticeably concave. Its shape is reminiscent of that of a parabolic dish antenna, except that its curvature is not typically reported to be symmetrical about the disk's center, but rather to be somewhat greater on its nearer (left) side. When the disk is seen in its true orientation, it always appears to be flat. The authors, plus a number of subjects and informal observers, were able to spontaneously report this effect, although some subjects did not report it, even after prompting. This effect may be related to the retinal motion gradient which exists, with the slowest retinal speeds of the dots near the center and progressively greater retinal speeds toward the periphery. The gradients of both retinal speed and size are more greatly compressed between the center and the left side than between the center and the right side due to the off-axis viewing angle. This may result in the asymmetrical, apparent concavity of the disk in its reversed orientation.

Second, the perceived size of the dots covering the standard disk changes very clearly during an orientation reversal. Those on the right side of the disk, which appears to be further away, appear to be larger than those on the left side, which appear to be closer to the subject. When the disk is perceived in its actual orientation, however, the dots on the right and left sides appear to be the same size, even though the retinal sizes of those on the right (nearer) side are greater than those on the left (farther) side.

Third, at the same time that the perceived sizes of the dots at the left and right sides of the rotating disk alter, their perceived speeds alter as well. The ones on the right are seen as moving faster than those on the left. But when the disk is perceived in its actual orientation, the dots on the left and right appear to be moving at the same speed.

Both the perceived size and speed changes which occur during the illusory depth orientation are consistent with constancy mechanisms involving perceived distance. Since the retinal sizes of the dots are constant, the reduction in their perceived size on the side that appears nearer and their increase in perceived size on the side that appears farther is consistent with the size-distance invariance hypothesis (Gogel, Wist, \& Harker, 1963; Kilpatrick \& Ittelson, 1953).

Furthermore, the decrease in their perceived speed on the perceptually nearer side during a reversal, as well as their increase in perceived speed on the perceptually farther side, is consistent with the evidence for a speed-constancy mechanism involving perceived distance (Wist, Diener, \& Dichgans, 1976).

In conclusion, we wish to stress the paradoxical character of this illusion again. It would be more 
comprehensible, even though iess interesting, if it occurred most readily with a textureless stationary or moving disk. ${ }^{2}$ in this case, the monocular retinal projection would be equally compatible with three different depth orientations: (1) the actual orientation, (2) an orientation at right angles to the actual orientation, ${ }^{3}$ or $(3$, an ellipse in the frontoparallel plane. We found, however, that Alternative 2 occurs only when the disk is in motion, that texture in the form of randomly distributed dots facilitates this alternative, and that Alternative 3 is rarely perceived. This is true in spite of the fact that the retinal size and motion gradient formed by the dots is consistent with Alternative 1, since the retinally largest and fastest dots are located at the physically nearer edge and the retinally smaller and slower ones are located at the physically farther edge. Thus, both the retinal size and speed gradients are most consistent with the actual orientation of the disk, but they nonetheless facilitated perception of reversed depth orientation. Why this is so remains an open question.

If a large projected speed difference between the center and periphery of the rotating disk is necessary in order for depth reversals to occur, it would be expected that with small disks, viewed at the same distance and orientation as the standard disk, depth reversals would be less apparent. With small disks, the difference in retinal speed between the center and periphery would be smaller. But, as mentioned earlier, we found the illusion to be strong with $10-\mathrm{cm}$ disks, which were $1 / 10$ th the diameter of the standard disk. It is not likely then, that the projected speed difference between center and periphery is critical in producing depth reversals. However, it is possible that rate of change in retinal speed from center to periphery, or the slope of the motion gradient, which is unaffected by the diameter of the disk may be the crucial variable. It was found that increasing the obliqueness of the viewing angle for the standard disk did increase the number of reversals per trials (Hunt et al., Note 2). But while this increased the slope of the motion gradient, it also made the retinal projection of the disk increasingly more elliptical. Further studies involving variations in the steepness of the motion gradient while holding the projected shape of the disk constant should resolve this issue.

\section{REFERENCE NOTE}

1. Hunt, B., Progin, T., \& Stone, J. Reversals in depth of a rotary stimulus. Unpublished manuscript, Franklin and Marshall College, 1976.

\section{REFERENCES}

Attneave, F. Multistability in perception. Scientific American, 1971, 225, 63-71.

Dichgans, J., Diener, H.-C., \& Brandt, Th. Optokineticograviceptive interaction in different head positions. Otolaryngologica, 1974, 78, 391-398.

Gogel, W. C., Wist, E. R., \& Harker, G. S. A test of the invariance of the ratio of perceived size to perceived distance. American Journal of Psychology, 1963, 76, 537-553.

Kilpatrick, F. P., \& Ittelson, W. H. The size-distance invariance hypothesis. Psychological Review, 1953, 60, 223-230.

Wist, E. R., Diener, H.-C., \& Dichgans, J. Motion constancy dependent upon perceived distance and the spatial frequency of the stimulus pattern. Perception \& Psychophysics, 1976, 19 , 485-491.

\section{NOTES}

1. Although the means for the standard disk for Groups 1 and 2 were combined in the table, the t tests were carried out using the separate group means. The Ns for Groups 1 and 2 are reduced by 1 and 2, respectively, in the table due to the deviant results of three subjects. The one dropped from the Group 1 analysis saw reversals for only 4 of the 10 stimuli presented. Of the two subjects dropped from the Group 2 analysis, one showed the most pronounced illusory effect we have observed to date: For this subject, the disk shifted to the illusory orientation after a relatively long latency and remained there for the rest of the 1 -min trial period. The data of the second subject was like that of the Group 1 subject in that reversals were seen for only 2 of the 8 stimuli presented. The inclusion of the data of these extreme subjects would have seriously distorted the mean data.

2. It should be noted that, while the "zero rings" stimulus in Panel $\mathrm{Cl}$ of the figure was nominally textureless, its surface was nonetheless rough enough so that its direction and speed of movement were readily discernible at the stimulus speed employed. However, if the speed was increased to about $300^{\circ} / \mathrm{sec}(50 \mathrm{rpm})$, the texture was not visible and the illusion failed to occur.

3. Although no quantitative data on the perceived angle of orientation of the disk during depth reversals were obtained, selected subjects were separately asked to indicate whether the disk appeared slanted in depth at an angle greater than, less than, or equal to right angles to the line of sight. They invariably reported the slant to be greater than $90^{\circ}$.

(Received for publication August 8, 1977; revision accepted January 24, 1978.) 